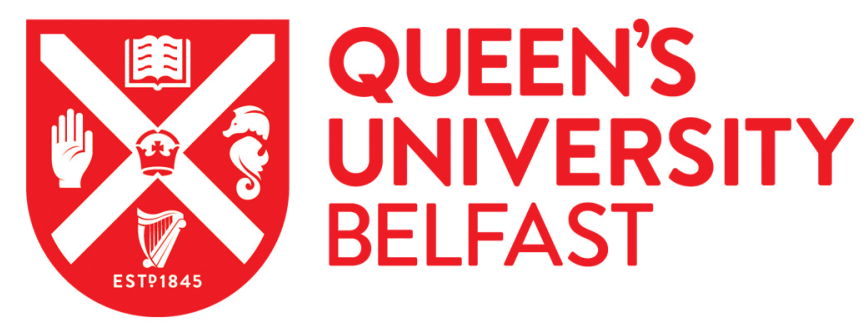

\title{
Association between overall fruit and vegetable intake, and fruit and vegetable sub-types and blood pressure: the PRIME study (Prospective Epidemiological Study of Myocardial Infarction)
}

Elsahoryi, N. A., Neville, C. E., Patterson, C. C., Linden, G. J., Moitry, M., Biasch, K., Kee, F., Amouyel, P., Bongard, V., Dallongeville, J., Ferrières, J., \& Woodside, J. V. (2020). Association between overall fruit and vegetable intake, and fruit and vegetable sub-types and blood pressure: the PRIME study (Prospective Epidemiological Study of Myocardial Infarction). The British journal of nutrition.

https://doi.org/10.1017/S0007114520001518

Published in:

The British journal of nutrition

Document Version:

Peer reviewed version

Queen's University Belfast - Research Portal:

Link to publication record in Queen's University Belfast Research Portal

Publisher rights

(c) The Authors 2020. This work is made available online in accordance with the publisher's policies. Please refer to any applicable terms of use of the publisher.

\section{General rights}

Copyright for the publications made accessible via the Queen's University Belfast Research Portal is retained by the author(s) and / or other copyright owners and it is a condition of accessing these publications that users recognise and abide by the legal requirements associated with these rights.

\section{Take down policy}

The Research Portal is Queen's institutional repository that provides access to Queen's research output. Every effort has been made to ensure that content in the Research Portal does not infringe any person's rights, or applicable UK laws. If you discover content in the Research Portal that you believe breaches copyright or violates any law, please contact openaccess@qub.ac.uk. 
Association between overall fruit and vegetable intake, and fruit and vegetable sub-types and blood pressure: the PRIME study (Prospective Epidemiological Study of Myocardial Infarction)

Nour A. Elsahoryi ${ }^{1 *}$, Charlotte E. Neville ${ }^{1 *}$, Christopher C. Patterson ${ }^{1}$, Gerry J. Linden ${ }^{1}$, Marie Moitry ${ }^{2}$, Katia Biasch ${ }^{2}$, Frank Kee ${ }^{1}$, Philippe Amouyel ${ }^{3}$, Vanina Bongard ${ }^{4}$, Jean Dallongeville $^{3}$, Jean Ferrières ${ }^{4}$, Jayne V. Woodside ${ }^{1}$

${ }^{1}$ Queen's University Belfast, Belfast, United Kingdom; ${ }^{2}$ MONICA-Strasbourg, Strasbourg, France; ${ }^{3}$ MONICA-Lille, Lille, France; ${ }^{4}$ MONICA-Toulouse, Toulouse, France.

*Joint first authors

Corresponding author: Professor Jayne Woodside, Institute for Global Food Security (Centre for Public Health), School of Medicine, Dentistry and Biomedical Sciences, Queen's University Belfast, Institute of Clinical Science A (First Floor), Grosvenor Road, Belfast, BT12 6BJ, UK, Tel: 00442890 978942, Fax: 00442890 235900, E-mail: j.woodside@qub.ac.uk

Shortened version of the title: Blood pressure and fruit and vegetables

Keywords: blood pressure, fruit and vegetable sub-types; dietary assessment; cardiovascular disease risk

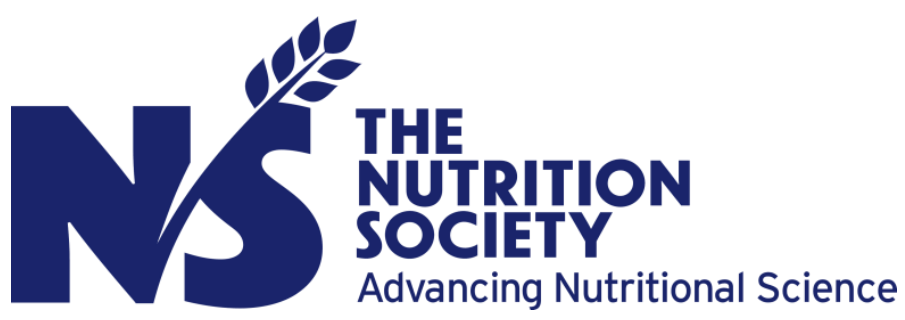

This peer-reviewed article has been accepted for publication but not yet copyedited or typeset, and so may be subject to change during the production process. The article is considered published and may be cited using its DOI

$10.1017 /$ S0007114520001518

The British Journal of Nutrition is published by Cambridge University Press on behalf of The Nutrition Society 


\section{Abstract}

Increased fruit and vegetable (FV) intake is associated with reduced blood pressure. However, it is not clear whether the effect of FV on blood pressure depends on the type of FV consumed. Furthermore, there is limited research regarding the comparative effect of juices or whole FV on blood pressure. Baseline data from a prospective cohort study examined the cross-sectional association between total FV intake, but also specific types of FV and blood pressure in France and Northern Ireland. A total of 10660 men aged 50-59 years were recruited from 1991 to 1994 . Blood pressure was measured in a clinic setting, and dietary intake was assessed by food-frequency questionnaire (FFQ). After adjusting for potential confounders, both systolic (SBP) and diastolic blood pressure (DBP) were significantly inversely associated with total fruit, vegetable and fruit juice intake however when results were examined according to the sub-type of fruit or vegetable (citrus fruit, other fruit, fruit juices, cooked vegetables and raw vegetables), only the other fruit and raw vegetable categories were consistently associated with reduced SBP and DBP. In relation to the risk of hypertension based on systolic blood pressure $>140 \mathrm{mmHg}$, the odds ratio for total fruit, vegetable and fruit juice intake (per fourth) was 0.95 (95\% CI 0.91, 1.00), with the same estimates being 0.98 (CI \% 0.94, 1.02) for citrus fruit intake (per fourth), 1.02 (CI \% $0.98,1.06$ ) for fruit juice intake (per fourth), 0.93 (CI \% 0.89, 0.98) for other fruit intake (per fourth), 1.05 (CI \% 0.99, 1.10) for cooked vegetable intake (per fourth) and 0.86 (CI \% 0.80, 0.91) for raw vegetable intake (per fourth). Similar results were obtained for DBP. In conclusion, a high overall intake of fruit, vegetables and fruit juice was inversely associated with SBP and DBP and risk of hypertension, but that this association differs by FV sub-type, suggesting that the strength of the association between these FV sub-types and blood pressure might be related to the type consumed, or to processing or cooking-related factors. 


\section{Introduction}

Hypertension is a major public health challenge and it is the most important, modifiable risk factor for cardiovascular diseases (CVD) incidence and mortality ${ }^{(1)}$. The global prevalence of hypertension reached $22 \%$ in 2014 and is expected to increase to $29.2 \%$ in 2025 if current trends persist ${ }^{(2)}$. Among US adults, the crude prevalence of hypertension was $45.6 \%$ and, according to the 2017 ACC/AHA guidelines, antihypertensive medication was recommended for $36.2 \%$ of the adult population, ${ }^{(3)}$. Lifestyle modification and dietary management can be an effective treatment for high blood pressure (BP), in addition to medication in advanced stages $^{(4)}$.

A high fruit and vegetable (FV) intake has been associated with reduced $\mathrm{BP}^{(5-8)}$ as well as a reduction in the risk of $\mathrm{CVD}^{(9-10)}$, including coronary heart disease $(\mathrm{CHD})^{(11)}$. A number of intervention studies have shown that increased daily intake of FV decreased BP significantly compared with a control diet $\left.{ }^{(12,13}\right)$. In contrast, some intervention studies have not demonstrated reductions in BP in response to increased FV intake ${ }^{(14-16)}$. These contrasting results may be related to variations in the type of FV consumed within each intervention, but whether the effect of FV on BP depends on the type of FV consumed is largely unknown. For example, FV juices, as a sub-type of FV, are generally thought to have less desirable effects than fresh FV because they contain less fibre, although similar levels of other nutrients, e.g. vitamin $\mathrm{C}^{(8,17)}$. The comparative effect of juices or whole FV on BP and other CVD outcomes is relatively understudied. There is also debate regarding the effect of cooked vegetables versus raw vegetables on health and the effect of processing/cooking on the nutritional content of FV and its effect on health, including CVD risk factors and hard CVD outcomes $^{(18)}$. For example, cross-sectional results from the INTERMAP study showed that both raw and cooked FV were associated with BP, with the association with raw vegetables being somewhat stronger than for cooked vegetables ${ }^{(18)}$.

This study aimed to determine the relationship between daily portions of FV intake, either considered overall or as specific sub-types of FV (citrus fruit, other fruit, fruit juices, cooked vegetables and raw vegetables) and BP in 50-59 year old men from France and Northern Ireland (NI). The overall hypothesis was that higher overall FV intake would be associated with reduced BP, but that the association may differ according to the type of FV consumed. 
Method

Study population

The PRIME (Prospective Epidemiological Study of Myocardial Infarction) study is a multicentre, prospective cohort study examining cardiovascular disease and associated risk factors in men. The study was initiated from previous collaborative work carried out within the WHO 'MONICA project' (Multinational Monitoring of trends and determinants in CVD) ${ }^{(19)}$. Sampling procedures, study design and primary endpoints have been described fully elsewhere $^{(19)}$. Between 1991 and 1994, 10,600 male participants were recruited, aged between 50-59 years, in four different centres (one centre in NI and three in France): Lille $(n=2,633)$, Strasbourg $(n=2,612)$, and Toulouse $(n=2,610)$ in France and Belfast $(n=2,745)$ in NI. The sample was recruited to broadly match the social class structure of the population. Written consent was obtained from all participants at baseline and ethical approval was obtained from the Research Ethics Committee of the Faculty of Medicine, Queen's University Belfast.

\section{Assessment of exposure measures}

Dietary data were collected via a short, self-administered 16-item FFQ at baseline, in the participant's home, and was later checked by an interviewer in the clinic for consistency and missing responses. Participants were asked to indicate their usual frequency of consumption of a standard portion of fruit or vegetable based on the last weeks using the following scale: more than once per d (number per d); daily; three to four times per week; once per week; twice per month; once per month; never. The FFQ measured the frequency of consumption of 16 food items including overall intake of FV and FV sub-types. For the purposes of this study, frequencies of intake were converted into portions per day. The FV were then grouped into five separate sub-types (citrus fruit, fruit juice, other fruit, raw vegetables and cooked vegetables), as asked within the FFQ, and also summed to provide a measure of overall/total fruit, vegetable and fruit and vegetable juice (FVJ) intake. Potatoes were not included as they are not considered to be a vegetable in the UK, and they have not been included in previous PRIME analyses ${ }^{(20)}$.

\section{Assessment of outcome measures}

All participants underwent a clinical examination at baseline to obtain anthropometric measurements including height (to the nearest $\mathrm{cm}$ ), weight (to the nearest 200g) and waist 
and hip circumference (to the nearest $0.5 \mathrm{~cm}$ ). All measurements were carried out using standardized instruments and procedures. Body mass index (BMI) was computed as weight $(\mathrm{kg})$ divided by height squared $\left(\mathrm{m}^{2}\right)$. Resting SBP and DBP was measured once at the end of the examination with an automatic device (Spengler SP9; Spengler, F94230 Cachan, France), by trained staff, after a five minute rest in the sitting position.

\section{Assessment of other variables}

At baseline, participants completed self-administered questionnaires relating to demographic and socioeconomic factors ${ }^{(19)}$. Participants then attended a clinic, where their questionnaires were checked for completeness. Information was collected on participants' socioeconomic status, psychosocial factors, medication and tobacco use, physical activity level and personal and family medical history ${ }^{(20)}$. Socio-economic status was based on a composite score of material conditions in the household based on three proxy indicators (the type of living accommodation (rented or owned/mortgage), the number of cars/vans/motorcycles in the household and the number of baths and/or showers and toilets in the home). Composite scores were categorised into low, medium and high ${ }^{(21)}$.

Lifetime smoking was categorised as: never smoked, smoked other than cigarettes, smoked $<15$ cigarette pack years, smoked $\geq 15$ but $<30$ cigarette pack years and smoked $\geq 30$ cigarette pack years. Physical activity was recorded in metabolic equivalent scores per week. A CVD screening examination was also conducted at baseline which included a detailed history of previous CVD and asked participants to report if a doctor had ever identified them as having a given risk factor for CVD and to state any past or current treatment. In addition to this, the London School of Hygiene CVD Questionnaire for Chest Pain on Effort and Possible Infarction was used for each participant ${ }^{(22)}$. Participants were also asked to give details on any history of diabetes: diabetes was defined by the current intake of oral hypoglycaemic treatment or use of insulin. Self-reported alcohol consumption was recorded in the form of a daily diary which participants completed over a 7 day period for a number of different types of alcoholic drinks. Alcohol intake was converted into $\mathrm{ml}$ per week and subsequently categorised as: none, 1-128, 129-265, 266-461 and $\geq 462 \mathrm{ml}$ per week.

Blood samples were drawn at baseline from each participant after a 12 hour fast. Venous blood was collected into EDTA tubes and returned to the local laboratory within four hours 
of collection ${ }^{(19)}$. Lipids, including total cholesterol and HDL cholesterol, were analysed immediately, while other samples were aliquoted for long-term storage at $-150{ }^{\circ} \mathrm{C}$.

Statistical methods

Statistical analysis was performed using SPSS v22.0 (SPSS Inc, Chicago, IL). Data were summarised as mean and SD. Chi-square and independent samples t-tests were used to compare categorical data and continuous data, respectively, between countries. Differences in general characteristics across quartile categories of FV intakes were also assessed using descriptive statistical tests. One-way ANOVA with Tukey's post-hoc comparison was used for continuous variables and chi-square test was used for categorical variables. Univariate linear and logistic regression models were used to examine associations between BP and overall FV and the five FV sub-types. For linear regression, systolic blood pressure (SBP) and diastolic blood pressure (DBP) were analysed as continuous variables and FV sub-types were analysed as categorical variables (i.e. per fourth). The following cut-offs (Q1 - Q4) were used: Citrus fruit $\leq 0.07,0.08-0.29,0.30-0.50, \geq 0.50$ portions/day; Fruit juice $\leq 0.00$, $0.01-0.07,0.08-0.50, \geq 0.50$; Other fruit $\leq 0.14,0.15-0.50,0.51-1.00, \geq 1.01$; Raw vegetables $\leq 0.29,0.30-0.50,0.51-1.00, \geq 1.01$; Cooked vegetables $\leq 0.29,0.30-0.50,0.51-1.00, \geq 1.01$; FVJ $\leq 1.60,1.61-2.30,2.31-3.57, \geq 3.58$. For logistic regression, all dependent and independent variables were analysed as categorical variables. For BP, the following cutpoints were used to define hypertension: SBP $\geq 140$ and $\mathrm{DBP} \geq 90 \mathrm{mmHg}$. All regression analyses were adjusted for potential confounding factors which included factors that were associated with SBP, DBP and FV intake in the current analysis and also other commonly known confounders that have been previously highlighted in the literature. Model 1 was unadjusted; Model 2 was adjusted for age and country; Model 3 was adjusted as for Model 2 plus BMI, height, smoking (five categories), physical activity, total cholesterol, HDL cholesterol, education level (primary, secondary, technical and high), material conditions (low, medium and high) as a measure of socio-economic position, alcohol intake (five categories), diabetes and CHD history. Further sensitivity analyses were also conducted to examine potential intermediary effects of BMI and total cholesterol and HDL. For all analyses, a $p$ value of $\leq 0.05$ was considered statistically significant. 


\section{Results}

Baseline characteristics of all PRIME participants and for participants in NI and France separately are shown in Table 1. There were significant differences in all baseline characteristics between the two countries, with the exception of SBP. Age, BMI, history of diabetes, smoking (all levels), education level (all levels), material conditions (all levels), and DBP were significantly higher in France while alcohol intake, physical activity and SBP were significantly higher in NI compared to France. Intakes of total FVJ, citrus fruit, other fruit and raw vegetables were significantly higher in France compared to NI (all $p<0.001$ ) while intakes of fruit juices and cooked vegetables were significantly higher in NI compared to France. Significant positive correlations were observed between intakes of FV across the various sub groups (all $\mathrm{p}<0.001$, data not shown).

Table 2 shows the characteristics of participants across the quartiles of total FVJ intakes. Results showed a significant difference in age, incidence of diabetes, alcohol intake, physical activity, smoking, education level, material conditions and blood pressure across the quartiles of FVJ intake.

Table 3 shows the association between SBP and FV intake, both unadjusted and adjusted for confounders. Model 1 showed that FVJ intake was significantly associated with SBP, and this remained significant when adjusted for age and country (Model 2), and in the fully adjusted model (Model 3). When FV categories were examined separately, increased citrus fruit intake was significantly associated with reduced SBP in both the unadjusted analyses and when adjusted for age and country. However, significance was lost in the fully adjusted model. An association was also evident between increased other fruit and raw vegetable intake and reduced SBP in all models. In contrast, intake of fruit juice and intake of cooked vegetables showed no association with SBP in all models. SBP decreased by $0.46 \mathrm{mmHg}$ as intake of FVJ increased (per fourth), by $0.63 \mathrm{mmHg}$ as intake of other fruit increased (per fourth) and by $1.29 \mathrm{mmHg}$ as intake of raw vegetables increased (per fourth), after adjustment for potential confounders.

Table 4 shows the association between DBP and FV intake. The unadjusted analysis showed that FVJ intake was significantly associated with DBP and this remained significant after adjusting for age and country, and also in the fully adjusted model. When FV categories were examined separately, other fruit and raw vegetables were significantly associated with SBP, 
and this remained significant when adjusted for age and country, and also in the fully adjusted model. Both citrus fruit intake and cooked vegetable intake were associated with reduced DBP in the unadjusted analyses and after adjusting for age and country, but the association became non-significant in the fully adjusted model. In contrast, fruit juice intake was not associated with DBP in all models. DBP decreased significantly, by $0.45 \mathrm{mmHg}$, as intake of FVJ increase (per fourth), by $0.56 \mathrm{mmHg}$ as intake of other fruit increased (per fourth) and by $1.01 \mathrm{mmHg}$ as intake of raw vegetables increased (per fourth), after adjustment for all confounders.

Table 5 shows the association between risk of hypertension (based on SBP >140 mmHg) and FV intake, both as overall FVJ intake and by separate FV categories. The odds ratio of increased SBP was significantly reduced as FVJ intake increased, and this was significant in both the unadjusted and adjusted analyses. A similar pattern was evident for intake of other fruits and intake of raw vegetables. For citrus fruit, associations were significant in model 1 (unadjusted) and in model 2 (adjusted for age and country), but statistical significance was lost in the fully adjusted model. In contrast, no association was observed between fruit juice or cooked vegetable intake and SBP. The risk of hypertension decreased by $5 \%$ as intake of FVJ increased per fourth, and decreased by $7 \%$ as other fruit and $14 \%$ as raw vegetable intake increased per fourth.

Table 6 shows the association between risk of hypertension (based on DBP $\geq 90 \mathrm{mmHg}$ ) and FV intake, both as overall FVJ intake and by separate FV categories. The odds ratio for having increased DBP was significantly reduced as FVJ intake increased, and this was significant in both the unadjusted and adjusted analyses. A similar pattern was evident for intakes of other fruits and intake of raw vegetables. For citrus fruit, significant associations were observed after adjusting for age and country, however significance was lost in the fully adjusted model. In contrast, no association was observed between fruit juice or cooked vegetable intake and DBP. The risk of increased DBP decreased by $6 \%, 10 \%$ and $17 \%$ as intakes of FVJ, other fruit and raw vegetables increased (per fourth), respectively.

Further exploratory analyses were conducted to examine potential intermediary effects of BMI and also HDL and total cholesterol. The results of this analysis did not alter the findings observed (data not shown). 


\section{Discussion}

Using data collected from populations in Northern Ireland and France, SBP and DBP were significantly inversely associated with intake of overall FVJ, but, when considering subtypes, were only associated with other fruit and raw vegetables. Increased intakes of the two sub-types were consistently associated with reduced BP, and reduced risk of hypertension. There was no association between increased intake of citrus fruit, fruit juice or cooked vegetables and either SBP, DBP, or risk of hypertension. Most previous studies have considered FV together, without taking into consideration the FV sub-types $\left.{ }^{(12,16,23}\right)$. Some studies also include juice within the overall FV variable, while others do not ${ }^{(24)}$. Unlike other studies, the current study considered intakes of FV sub-types, as well as overall FVJ intake, with adjustment for confounders.

These results are in line with a number of studies that reported an inverse association between overall $\mathrm{FV}$ intake and $\mathrm{BP}^{(25)}$. A recent meta-analysis of observational studies concluded that increasing FV intake was associated with reduced $\mathrm{BP}^{(26)}$. Individual studies, not included in the meta-analyses, also reported similar findings ${ }^{(27-29)}$. The beneficial effect of overall FV intake on BP reduction is suggested to be due to the effect of the combination of nutrients and other components found in FV (for example, fibre, antioxidants, other vitamins and minerals) potentially acting synergistically to improve the vascular phenotype ${ }^{(12)}$. Determining the effect of any single nutrient within FV over other nutrients is very difficult, but examining the effect of overall FV as a food group may be more reflective and relevant to our habitual $\operatorname{diet}^{(12)}$. Our results support the notion that total FVJ intake has a protective effect against hypertension, likely due to the effect of the combination of many nutrients such as phytochemicals, vitamins and minerals ${ }^{(30)}$.

Analysis of FV sub-types indicated that other fruit (not citrus and not fruit juice) and raw vegetables were significantly associated with BP. In contrast, citrus fruit, fruit juice and cooked vegetables were not associated with BP outcomes. There is limited research on the association between sub-types of FV such as citrus fruit and BP. Our results are consistent with other studies which reported no association between fruit juice and $\mathrm{BP}^{(31-33)}$. As suggested by previous studies, this lack of association is possibly due to the low fibre and high sugar (either as added sugar or fructose) content ${ }^{(8,18)}$ which have been associated with high $\mathrm{BP}^{(34)}$. Conflicting evidence, however, comes from a number of previous studies which examined the effect of a single type of fruit juice on BP, with some reporting positive effects 
of specific types of fruit juices e.g. cherry juice, berry juice, pomegranate juice on $\mathrm{BP}^{(32,35,36)}$. It is possible that the specific type of fruit juice may be important in terms of its effect on BP, and may relate to the presence of other bioactive compounds ${ }^{(33)}$, or processing conditions ${ }^{(37)}$.

Interestingly, our findings showed a negative association between other fruit (not citrus and not fruit juice) and BP outcomes. Some studies have found inverse associations between single types of fruit, such as apple and tart cherry, and $\mathrm{BP}^{(8,32)}$. For example, Keane et al. concluded that Montmorency tart cherry intake acutely reduced SBP in men with early hypertension $^{(32)}$ while Oude Griep et al reported a positive relationship between DBP and apple intake in East Asian consumers, although this was not found in other countries ${ }^{(8)}$.

Finally, when comparing the association of cooked vegetables versus raw vegetables on BP, our results indicated a significant association between raw vegetable intake and BP, but no significant association between cooked vegetables and BP, after adjusting for confounding factors. The lack of association between cooked vegetables and BP in our study may be explained by the effect of the cooking method on the nutritional value of the vegetables. Similar findings were reported in the cross-sectional, US-based INTERMAP study which was conducted in 2195 males and females age 40-59 years. In this study an inverse association was noted between both raw vegetable intake and BP, and cooked vegetable intake and BP, but the association was stronger for raw vegetables ${ }^{(16)}$. The results of this study were potentially explained by the effect of cooking, which could significantly change the chemical composition of vegetables and influence the concentration and bioavailability of bioactive compounds, such as antioxidant, water-soluble and heat sensitive nutrients ${ }^{(38)}$. The effect also depends on cooking conditions (such as cooking duration and method) and morphological and nutritional characteristics of vegetable species, in addition to the interaction with other dietary factors that can affect nutrient absorption ${ }^{(38,39)}$.

\section{Study Strengths and Limitations}

Strengths of the current study are that it considered the sub-type of FV as well as overall FV intake. The analysis was also carried out, using the same methodology, on pooled data collected in France and Northern Ireland, two countries with significant differences in lifestyle behaviours. In addition, unlike other studies, the PRIME study sample was large and included a wide range of confounding factors. 
Limitations of the current analysis include the specific age group and gender of the population (males, aged 50-59 years), therefore it is difficult to generalise the findings to women or younger age groups. In addition, assessment of lifestyle behaviours relied on selfreport rather than objective measures. The use of a non-validated FFQ to assess dietary intake is also a limitation. Although, widely used in epidemiological studies, FFQs are prone to recall bias, thus limiting their accuracy in assessing dietary intake. In the current study, a short 16-item FFQ was used to assess dietary intake and the number of specific types of FV and fruit juices, therefore, further exploration of the association between further sub-types of FV and BP was not possible. Similarly, detailed information about vegetable cooking methods and processing were not available. However, previous results by Dauchet et al demonstrated that this FFQ was suitable for discriminating between low and high consumers of FV. In their analysis, they noted strong positive correlations between the self-reported FV intakes from the FFQ and biomarkers of FV status, specifically B-cryptoxanthin, vitamin C and $\alpha$ - and $\beta$-carotene ${ }^{20}$. Furthermore, a previous study reported that dietary questionnaires with restricted number of items do not overly affect the ability to rank individuals according to their FV intake ${ }^{40}$. However, we cannot rule out the possibility that the observed associations between FV intakes may be explained by compensatory changes in other food intakes that we were unable to explore. The retrospective nature of the FFQ is also a limiting factor in that it only reflected food consumption over the previous seven day period and therefore did not capture potential seasonal variation in food intake. Although our analyses were adjusted for country, there may also have been differences between the two countries in terms of overall dietary pattern. Indeed, a further analysis showed that when the two countries were analysed separately, the findings became stronger for France while those for Northern Ireland became attenuated, however, for Northern Ireland, this may simply have been due to lack of statistical power. The assessment of blood pressure also had limitations in that only one BP measurement was performed, therefore, results should be viewed with caution. Given the high variability of general BP measures, the use of one blood pressure measurement limits identification of cases of hypertension and in particular, limits continuous analyses with blood pressure. In addition, lifestyle behaviours were only assessed at one time-point, and data collection for this study took place from 1991-1993; therefore we cannot rule out the possibility of change in lifestyle behaviours, including dietary habits and food products consumed over time. The cross-sectional design of the study is also a key limitation in that both the exposure and outcome measures were simultaneously assessed, thus ruling out evidence of a temporal relationship. The cross-sectional design raises the issue of reverse 
causality. Indeed, the associations observed in the current study do by no means indicate, nor prove, that FV reduce blood pressure or hypertension. Reverse causality may weaken any true association between FV intake and blood pressure. Without longitudinal data, it is not possible to establish a true cause and effect relationship. It is also possible that other confounding factors not accounted for in the current study may be masking the true effect of FV intake on blood pressure. Finally, while the results are interesting, the lack of validation of the FFQ together with the limited assessment of BP means that the overall results need to be interpreted with caution. This cohort will have included men at baseline who had been diagnosed with hypertension and were being managed by anti-hypertensive medication. These participants may have been classified as non-hypertensive, but that will have been due to the prescribing of anti-hypertensive medication and the control of their BP. In the current study, we were unable to adjust for use of BP medication due to the lack of availability of anti-hypertensive medication data for the French cohort. However, reanalysis of the Belfast cohort with exclusion of those who reported use of anti-hypertensive medication at baseline did not alter findings (data not shown).

\section{Conclusion}

In conclusion, after adjusting for potential confounding factors, the results of cross-sectional analysis suggested that overall FVJ intake may be associated with reduced BP and reduced risk of hypertension. When FV were analysed separately, the association with BP depended on the FV sub-type, with other fruit and raw vegetable intake being inversely associated with BP, but not fruit juice, citrus fruit or cooked vegetables. These results suggest that the strength of the association between FV sub-types and BP might be related to the type of FV consumed, or to processing or cooking-related factors. A more defined classification of FV consumed during dietary data collection may provide more valuable information when studying associations with health outcomes. Further intervention studies to examine the dose-response effects of specific FV on BP are recommended, with a consideration of the possible effect of factors, such as storage, processing and cooking, that will impact on overall nutrient profile.

\section{Acknowledgments}

The PRIME Study is organised under an agreement between INSERM and the Merck, Sharpe and Dohme- Chibret Laboratory, with the following participating Laboratories:

The Strasbourg MONICA Project, Strasbourg, France (M.Moitry, D. Arveiler, B. Haas). 
The Toulouse MONICA Project, INSERM U558, Toulouse, France (J. Ferrières, JB. Ruidavets).

The Lille MONICA Project, INSERM U744, Lille, France (P. Amouyel, M. Montaye).

The Department of Epidemiology and Public Health, Queen's University Belfast, NI (A. Evans, J. Yarnell, F.Kee).

The Department of Atherosclerosis, INSERM U545, Lille, France (G. Luc, JM. Bard).

The Laboratory of Hematology, La Timone Hospital, Marseille, France (I. Juhan-Vague).

The Laboratory of Endocrinology, INSERM U326, Toulouse, France (B. Perret).

The Vitamin Research Unit, The University of Bern, Bern, Switzerland (F. Gey).

The Trace Element Laboratory, Department of Medicine, Queen's University Belfast, NI (J. Woodside, I. Young).

The DNA Bank, INSERM U525, Paris, France (F. Cambien).

The Coordinating Center, INSERM U909, Villejuif, France (P. Ducimetière, A. Bingham).

\section{Financial Support}

The present analysis was financially supported by University of Petra (UOP), Jordan. info@uop.edu.jo

\section{Conflict of Interest}

None

\section{Authorship}

The authors' responsibilities were as follows: NAE prepared the initial draft of the manuscript and carried out the statistical analysis; CCP directed the statistical analysis; CEN assisted with statistical analysis and drafting the manuscript; FK and GJL were coinvestigators of the Belfast centre of the PRIME study; MM and KB were co-investigators of the Strasbourg centre of the PRIME study; PA and JD were co-investigators of the Lille centre of the PRIME study; VB and JF were co-investigators of the Toulouse centre of the PRIME study. All of the authors contributed to the drafts, revisions and proof reading of the manuscript. JVW had the primary responsibility for the final content. 


\section{References}

1. Kjeldsen SE. (2018) Hypertension and cardiovascular risk: General aspects. Pharmacol Res 129, 95-99.

2. Kearney PM, Whelton M, Reynolds K et al. (2005) Global burden of hypertension: analysis of worldwide data. Lancet $\mathbf{3 6 5}, 217-23$.

3. Muntner P, Carey RM, Gidding S et al. (2018) Potential US Population Impact of the 2017 ACC/AHA High Blood Pressure Guideline. Circulation 137, 109-18.

4. NICE Guidelines. (2011) Hypertension in adults: diagnosis and management. Natl Inst Heal Care Excell.

5. Wang L, Manson JE, Gaziano JM et al. (2012) Fruit and vegetable intake and the risk of hypertension in middle-aged and older women. Am J Hypertens 25, 180-9.

6. Tsubota-Utsugi M, Ohkubo T, Kikuya M et al. (2011) High fruit intake is associated with a lower risk of future hypertension determined by home blood pressure measurement: the OHASAMA study. J Hum Hypertens 25, 164-71.

7. Nuñez-Cordoba JM, Alonso A, Beunza JJ, et al. (2009) Role of vegetables and fruits in Mediterranean diets to prevent hypertension. Eur J Clin Nutr 63, 605-12.

8. Oude Griep LM, Stamler J, Chan Q et al. (2013) Association of raw fruit and fruit juice consumption with blood pressure: the INTERMAP Study. Am J Clin Nutr 97, 1083-91.

9. Scheffers FR, Boer JMA, Verschuren WMM, et al. (2019) Pure fruit juice and fruit consumption and the risk of CVD: the European Prospective Investigation into Cancer and Nutrition-Netherlands (EPIC-NL) study. Br J Nutr 121, 351-359.

10. Miller V, Mente A, Dehghan M, et al. (2017) Fruit, vegetable, and legume intake, and cardiovascular disease and deaths in 18 countries (PURE): a prospective cohort study. Lancet, 390, 2037-2049.

11. Dauchet L, Amouyel P, Hercberg S et al. (2006) Fruit and vegetable consumption and risk of coronary heart disease: a meta-analysis of cohort studies. J Nutr 136, 2588-93.

12. Appel LJ, Moore TJ, Obarzanek E et al. (1997) A clinical trial of the effects of dietary patterns on blood pressure. DASH Collaborative Research Group. N Engl J Med 336, 
$1117-24$.

13. Appel LJ, Champagne CM, Harsha DW et al. (2003) Effects of comprehensive lifestyle modification on blood pressure control: main results of the PREMIER clinical trial. JAMA 289, 2083-93.

14. Berry SE, Mulla UZ, Chowienczyk PJ et al. (2010) Increased potassium intake from fruit and vegetables or supplements does not lower blood pressure or improve vascular function in UK men and women with early hypertension: a randomised controlled trial. Br J Nutr 104, 1839-47.

15. Broekmans WM, Klöpping-Ketelaars WA, Kluft C et al. (2001) Fruit and vegetables and cardiovascular risk profile: a diet controlled intervention study. Eur J Clin Nutr $55,636-42$.

16. McCall DO, McGartland CP, McKinley MC et al. (2009) Dietary intake of fruits and vegetables improves microvascular function in hypertensive subjects in a dosedependent manner. Circulation 119, 2153-60.

17. Liu K, Xing A, Chen K et al. (2013) Effect of fruit juice on cholesterol and blood pressure in adults: a meta-analysis of 19 randomized controlled trials. PloS One 8, 61420.

18. Chan Q, Stamler J, Brown IJ et al. (2014) Relation of raw and cooked vegetable consumption to blood pressure: the INTERMAP Study. J Hum Hypertens 28, 353-9.

19. Yarnell JW. (1998) The PRIME study: classical risk factors do not explain the severalfold differences in risk of coronary heart disease between France and Northern Ireland. Prospective Epidemiological Study of Myocardial Infarction. QJM 91, 66776.

20. Dauchet L, Ferrières J, Arveiler D et al. (2004) Frequency of fruit and vegetable consumption and coronary heart disease in France and Northern Ireland: the PRIME study. Br J Nutr 92, 963-72.

21. Wagner A, Simon C, Evans A et al. (2003) Physical activity patterns in 50-59 year men in France and Northern Ireland. Associations with socio-economic status and health behaviour. Eur J Epidemiol 18, 321-9.

22. Rose GA, Blackburn H, Gillum RF et al. (1982) Cardiovascular Survey Methods, 2nd 
Edition. 2nd ed. Monograph Series. 56. Geneva, Switzerland: WHO

23. Moore TJ, Conlin PR, Ard J et al. (2001) DASH (Dietary Approaches to Stop Hypertension) diet is effective treatment for stage 1 isolated systolic hypertension. Hypertension 38, 155-8.

24. Hills M, Armitage P. (1979) The two-period cross-over clinical trial. Br J Clin Pharmacol 8, 7-20.

25. Woodside J V, Young IS, McKinley MC. (2013) Fruit and vegetable intake and risk of cardiovascular disease. Proc Nutr Soc 72, 399-406.

26. Li B, Li F, Wang L et al. (2016) Fruit and vegetables consumption and risk of hypertension: a meta-analysis. J Clin Hypertens 18, 468-76.

27. Ascherio A, Hennekens C, Willett WC et al. (1979) Prospective study of nutritional factors, blood pressure, and hypertension among US women. Hypertens 27, 1065-72.

28. Dauchet L, Kesse-Guyot E, Czernichow S et al. (2017) Dietary patterns and blood pressure change over 5-y follow-up in the SU.VI.MAX cohort. Am J Clin Nutr 85, 1650-6.

29. Steffen LM, Kroenke CH, Yu X et al. (2005) Associations of plant food, dairy product, and meat intakes with 15-y incidence of elevated blood pressure in young black and white adults: the Coronary Artery Risk Development in Young Adults (CARDIA) Study. Am J Clin Nutr 82, 1169-77.

30. Srinath Reddy K, Katan MB. (2004) Diet, nutrition and the prevention of hypertension and cardiovascular diseases. Public Health Nutr 7(1A), 167-86.

31. Kivimäki AS, Siltari A, Ehlers PI et al (2013). Lingonberry juice lowers blood pressure of spontaneously hypertensive rats (SHR). J Funct Foods 5, 1432-40.

32. Keane KM, George TW, Constantinou CL et al (2016). Effects of Montmorency tart cherry (Prunus Cerasus L.) consumption on vascular function in men with early hypertension. Am J Clin Nutr 103, 1531-9.

33. Sahebkar A, Ferri C, Giorgini P et al (2017). Effects of pomegranate juice on blood pressure: A systematic review and meta-analysis of randomized controlled trials. Pharmacol Res 115, 149-61. 
34. Pase MP, Grima N, Cockerell R et al (2015). Habitual intake of fruit juice predicts central blood pressure. Appetite 84, 68-72.

35. Seshadri S, Beiser A, Kelly-Hayes M et al. (2006) The lifetime risk of stroke: estimates from the Framingham Study. Stroke 37, 345-50.

36. Tjelle TE, Holtung L, Bøhn SK et al (2015). Polyphenol-rich juices reduce blood pressure measures in a randomised controlled trial in high normal and hypertensive volunteers. Br J Nutr 114, 1054-63.

37. Wallace IR, McEvoy CT, Hunter SJ et al. (2013) Dose-response effect of fruit and vegetables on insulin resistance in people at high risk of cardiovascular disease: a randomized controlled trial. Diabetes Care 36, 3888-96.

38. Miglio C, Chiavaro E, Visconti A et al. (2008) Effects of different cooking methods on nutritional and physicochemical characteristics of selected vegetables. J Agric Food Chem 56, 139-47.

39. Jiménez-Monreal AM, García-Diz L, Martínez-Tomé M et al. (2009) Influence of cooking methods on antioxidant activity of vegetables. J Food Sci 74, H97-103.

40. Thompson FE, Subar AF, Smith AF, et al. (2002) Fruit and vegetable assessment: performance of 2 new short instruments and a food frequency questionnaire. J Am Diet Assoc 102, 1764-1772. 
Table 1. Baseline characteristics of participants in the PRIME study

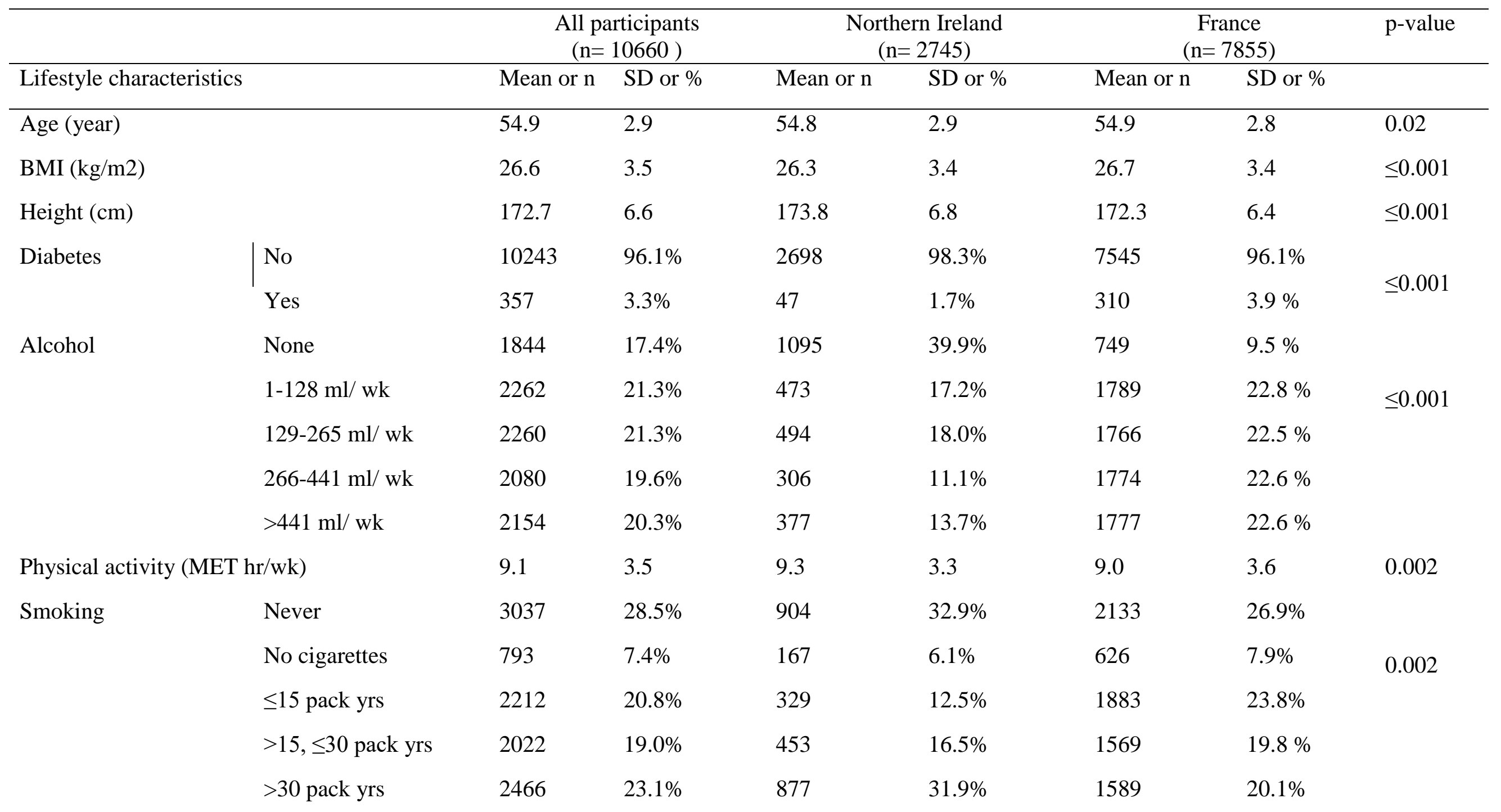


Accepted manuscript

\begin{tabular}{|c|c|c|c|c|c|c|c|c|}
\hline \multirow[t]{4}{*}{ Education Level } & Primary & 2412 & $22.6 \%$ & 743 & $27.1 \%$ & 1669 & $21.1 \%$ & \multirow{4}{*}{$\leq 0.001$} \\
\hline & Secondary & 1203 & $11.3 \%$ & 391 & $14.2 \%$ & 812 & $10.3 \%$ & \\
\hline & Technical & 3486 & $32.7 \%$ & 855 & $31.1 \%$ & 2631 & $33.2 \%$ & \\
\hline & Higher & 3179 & $29.8 \%$ & 690 & $25.1 \%$ & 2489 & $31.4 \%$ & \\
\hline \multirow[t]{3}{*}{ Material conditions } & Low & 2656 & $24.9 \%$ & 1075 & $39.2 \%$ & 1581 & $20.0 \%$ & \multirow{3}{*}{$\leq 0.001$} \\
\hline & Medium & 1601 & $15.0 \%$ & 586 & $21.3 \%$ & 1015 & $12.8 \%$ & \\
\hline & High & 6304 & $59.1 \%$ & 1081 & $39.4 \%$ & 5223 & $66.0 \%$ & \\
\hline $\mathrm{SBP}(\mathrm{mm} \mathrm{Hg})$ & & 133.7 & 19.0 & 133.9 & 20.6 & 133.7 & 18.4 & 0.54 \\
\hline $\mathrm{DBP}(\mathrm{mm} \mathrm{Hg})$ & & 83.65 & 11.69 & 81.74 & 11.5 & 84.32 & 11.69 & $\leq 0.001$ \\
\hline \multirow[t]{6}{*}{ FVJ intake (portions/d) } & FVJ & 2.60 & 1.40 & 2.28 & 1.40 & 2.70 & 1.37 & $<0.001$ \\
\hline & Citrus fruit & 0.45 & 0.52 & 0.36 & 0.47 & 0.47 & 0.53 & $<0.001$ \\
\hline & Fruit juice & 0.28 & 0.38 & 0.37 & 0.41 & 0.25 & 0.36 & $<0.001$ \\
\hline & Other fruit & 0.68 & 0.64 & 0.56 & 0.58 & 0.72 & 0.66 & $<0.001$ \\
\hline & Cooked vegetables & 0.63 & 0.46 & 0.71 & 0.54 & 0.60 & 0.43 & $<0.001$ \\
\hline & Raw vegetables & 0.56 & 0.46 & 0.28 & 0.44 & 0.66 & 0.43 & $<0.001$ \\
\hline
\end{tabular}


Table 2: Characteristics of participants across quartiles of total fruit, vegetable and juice intake

\begin{tabular}{|c|c|c|c|c|c|c|c|c|c|c|}
\hline \multirow[b]{2}{*}{ Characteristic } & & \multicolumn{2}{|l|}{ Q1 } & \multicolumn{2}{|l|}{ Q2 } & \multicolumn{2}{|l|}{ Q3 } & \multicolumn{2}{|l|}{ Q4 } & \multirow[t]{2}{*}{ p-value } \\
\hline & & Mean or $n$ & $\mathrm{SD}$ or $\%$ & Mean or $n$ & $\mathrm{SD}$ or $\%$ & Mean or $n$ & $\mathrm{SD}$ or $\%$ & Mean or $n$ & $\mathrm{SD}$ or $\%$ & \\
\hline Age (years) & & 54.76 & 2.90 & 54.86 & 2.89 & 54.96 & 2.88 & 55.06 & 2.86 & 0.002 \\
\hline BMI (kg/m2) & & 26.56 & 3.62 & 26.71 & 3.41 & 26.61 & 3.39 & 26.57 & 3.46 & 0.40 \\
\hline Height $(\mathrm{cm})$ & & 172.4 & 6.51 & 172.7 & 6.64 & 172.7 & 6.45 & 172.8 & 6.69 & 0.11 \\
\hline \multirow[t]{2}{*}{ Diabetes } & No & 2596 & $97.1 \%$ & 2316 & $97.1 \%$ & 3165 & $95.8 \%$ & 2083 & $96.7 \%$ & 0.02 \\
\hline & Yes & 76 & $2.8 \%$ & 70 & $2.9 \%$ & 138 & $4.2 \%$ & 71 & $3.3 \%$ & \\
\hline \multirow[t]{5}{*}{ Alcohol } & None & 486 & $18.2 \%$ & 408 & $17.1 \%$ & 546 & $16.5 \%$ & 389 & $18.0 \%$ & $<0.001$ \\
\hline & $1-128 \mathrm{ml} / \mathrm{wk}$ & 430 & $16.1 \%$ & 493 & $20.7 \%$ & 745 & $22.5 \%$ & 577 & $26.8 \%$ & \\
\hline & $129-265 \mathrm{ml} / \mathrm{wk}$ & 490 & $18.3 \%$ & 489 & $20.5 \%$ & 772 & $23.4 \%$ & 500 & $23.2 \%$ & \\
\hline & $266-441 \mathrm{ml} / \mathrm{wk}$ & 531 & $19.9 \%$ & 498 & $20.9 \%$ & 655 & $19.8 \%$ & 378 & $17.5 \%$ & \\
\hline & $>441 \mathrm{ml} / \mathrm{wk}$ & 735 & $27.5 \%$ & 498 & $20.9 \%$ & 585 & $17.7 \%$ & 310 & $14.4 \%$ & \\
\hline $\begin{array}{l}\text { Physical activity } \\
\text { (MET hr/wk) }\end{array}$ & & 8.91 & 3.72 & 8.92 & 3.47 & 9.19 & 3.44 & 9.18 & 3.52 & 0.003 \\
\hline \multirow[t]{5}{*}{ Smoking } & Never & 620 & $23.4 \%$ & 679 & $28.7 \%$ & 1036 & $31.5 \%$ & 685 & $31.9 \%$ & $<0.001$ \\
\hline & No cigarettes & 169 & $6.4 \%$ & 177 & $7.5 \%$ & 252 & $7.7 \%$ & 188 & $8.8 \%$ & \\
\hline & $\leq 15$ pack yr & 477 & $18.0 \%$ & 484 & $20.4 \%$ & 709 & $21.6 \%$ & 524 & $24.4 \%$ & \\
\hline & $>15, \leq 30$ pack yr & 519 & $19.6 \%$ & 459 & $19.4 \%$ & 628 & $19.1 \%$ & 396 & $18.5 \%$ & \\
\hline & $>30$ pack yr & 864 & $32.6 \%$ & 568 & $24.0 \%$ & 658 & $20.0 \%$ & 353 & $16.4 \%$ & \\
\hline \multirow{4}{*}{ Education level } & Primary & 761 & $29.3 \%$ & 495 & $21.3 \%$ & 675 & $21.0 \%$ & 455 & $21.9 \%$ & $<0.001$ \\
\hline & Secondary & 320 & $12.3 \%$ & 275 & $11.9 \%$ & 372 & $11.6 \%$ & 230 & $11.1 \%$ & \\
\hline & Technical & 932 & $35.9 \%$ & 827 & $35.7 \%$ & 1100 & $34.2 \%$ & 597 & $28.7 \%$ & \\
\hline & Higher & 584 & $22.5 \%$ & 712 & 30.7 & 1066 & $33.2 \%$ & 797 & $38.3 \%$ & \\
\hline \multirow[t]{3}{*}{ Material conditions } & Low & 896 & $33.6 \%$ & 566 & $23.8 \%$ & 750 & $22.8 \%$ & 411 & $19.2 \%$ & $<0.001$ \\
\hline & Medium & 426 & $16.0 \%$ & 376 & $15.8 \%$ & 488 & $14.8 \%$ & 302 & $14.1 \%$ & \\
\hline & High & 1342 & $50.4 \%$ & 1436 & $60.4 \%$ & 2056 & $62.4 \%$ & 1427 & $66.7 \%$ & \\
\hline SBP (mmHg) & & 134.96 & 20.34 & 133.83 & 18.53 & 133.51 & 18.32 & 132.32 & 18.53 & $<0.001$ \\
\hline $\mathrm{DBP}(\mathrm{mmHg})$ & & 84.30 & 12.28 & 83.73 & 11.33 & 83.58 & 11.44 & 82.80 & 11.62 & $<0.001$ \\
\hline
\end{tabular}

BMI, body mass index. SBP, systolic blood pressure. Continuous variables are presented as mean (SD) while categorical variables are presented as $\mathrm{n}(\%)$. Difference between fourths analysed using one way ANOVA for continuous variables and chi-square test for categorical variables 
Table 3. Linear regression analysis of the association between systolic blood pressure and FV variables in the PRIME study

\begin{tabular}{|c|c|c|c|c|c|c|}
\hline & FVJ & Citrus fruit & Fruit juices & Other fruit & Cooked vegetables & Raw vegetables \\
\hline \multicolumn{7}{|l|}{ Model 1} \\
\hline Q1 & 0.0 (reference) & 0.0 (reference) & 0.0 (reference) & 0.0 (reference) & 0.0 (reference) & 0.0 (reference) \\
\hline Q2 & $-1.13(-2.18,-0.08)$ & $-1.26(-2.20,-0.33)$ & $-1.84(-2.79,-0.89)$ & $-1.33(-2.29,-0.38)$ & $-0.45(-1.35,0.46)$ & $-0.64(-1.58,0.30)$ \\
\hline Q3 & $-1.45(-2.42,-0.49)$ & $-1.54(-2.68,-0.40)$ & $-1.12(-2.16,-0.07)$ & $-1.09(-2.04,-0.13)$ & $-0.19(-1.12,0.74)$ & $-2.53(-3.58,-1.49)$ \\
\hline Q4 & $-2.64(-3.72,-1.56)$ & $-1.12(-2.10,-0.14)$ & $-0.76(-1.76,0.23)$ & $-3.83(-5.21,-2.44)$ & $-1.39(-3.45,0.68)$ & $-4.39(-6.51,-2.27)$ \\
\hline Per Fourth & $-0.81(-1.15,-0.48)$ & $-0.36(-0.68,-0.05)$ & $-0.28(-0.59,0.03)$ & $-0.87(-1.26,-0.49)$ & $-0.18(-0.60,0.24)$ & $-1.39(-1.85,-0.93)$ \\
\hline p-value & $\leq 0.001$ & 0.02 & 0.08 & $\leq 0.001$ & 0.40 & $\leq 0.001$ \\
\hline \multicolumn{7}{|l|}{ Model 2} \\
\hline Q1 & 0.0 (reference) & 0.0 (reference) & 0.0 (reference) & 0.0 (reference) & 0.0 (reference) & 0.0 (reference) \\
\hline Q2 & $-1.21(2.24,-0.17)$ & $-1.10(-2.02,-0.17)$ & $-1.52(-2.46,-0.58)$ & $-1.27(-2.22,-0.33)$ & $-0.41(-1.31,0.48)$ & $-0.94(-1.94,0.06)$ \\
\hline Q3 & $-1.61(2.58,-0.65)$ & $-1.50(-2.63,-0.36)$ & $-0.89(-1.92,0.15)$ & $-1.22(-2.17,-0.26)$ & $-0.32(-1.26,0.62)$ & $-2.96(-4.11,-1.80)$ \\
\hline Q4 & $-2.88(-3.95,-1.80)$ & $-1.16(-2.14,-0.19)$ & $-0.78(-1.78,0.22)$ & $-4.15(-5.53,-2.76)$ & $-1.52(-3.57,0.53)$ & $-4.92(-7.09,-2.76)$ \\
\hline Per Fourth & $-0.89(-1.23,-0.56)$ & $-0.39(-0.70,-0.07)$ & $-0.27(-0.58,0.04)$ & $-0.97(-1.35,-0.58)$ & $-0.25(-0.67,0.18)$ & $-1.62(-2.12,-1.11)$ \\
\hline p-value & $\leq 0.001$ & 0.02 & 0.09 & $\leq 0.001$ & 0.25 & $\leq 0.001$ \\
\hline \multicolumn{7}{|l|}{ Model 3} \\
\hline Q1 & 0.0 (reference) & 0.0 (reference) & 0.0 (reference) & 0.0 (reference) & 0.0 (reference) & 0.0 (reference) \\
\hline Q2 & $-0.84(-1.89,0.21)$ & $-0.55(-1.49,0.38)$ & $-0.54(-1.49,0.41)$ & $-0.93(-1.89,0.04)$ & $-0.31(-1.20,0.59)$ & $-0.95(-1.98,0.07)$ \\
\hline Q3 & $-0.93(-1.91,0.05)$ & $-0.78(-1.92,0.36)$ & $-0.23(-1.28,0.82)$ & $-0.77(-1.74,0.21)$ & $0.33(-0.62,1.28)$ & $-2.36(-3.54,-1.18)$ \\
\hline Q4 & $-1.51(-2.62,-0.41)$ & $-0.15(-1.14,0.84)$ & $-0.29(-1.30,0.72)$ & $-2.82(-4.23,-1.41)$ & $-0.44(-2.52,1.64)$ & $-4.16(-6.33,-2.00)$ \\
\hline Per Fourth & $-0.46(-0.81,-0.12)$ & $-0.06(-0.38,0.26)$ & $-0.09(-0.41,0.23)$ & $-0.63(-1.03,-0.24)$ & $0.097(-0.33,0.53)$ & $-1.29(-1.81,-0.78)$ \\
\hline p- value & 0.01 & 0.72 & 0.59 & 0.002 & 0.66 & $\leq 0.001$ \\
\hline
\end{tabular}

$\mathrm{FV}$, fruit and vegetables. FVJ, fruit, vegetables and juice

Values represent mean difference $(95 \% \mathrm{CI})$ from reference category $(\mathrm{Q} 1)$

Model 1 unadjusted; Model 2 adjusted for age, country; Model 3 adjusted for age, country, cholesterol, BMI, height, physical activity, alcohol intake, education level, material conditions, smoking, diabetes and CHD history. 
Table 4. Linear regression analysis of the association between diastolic blood pressure and FV variables in the PRIME study

\begin{tabular}{|c|c|c|c|c|c|c|}
\hline & FVJ & Citrus fruit & Fruit juices & Other fruit & Cooked vegetables & Raw vegetables \\
\hline \multicolumn{7}{|l|}{ Model 1} \\
\hline Q1 & 0.0 (reference) & 0.0 (reference) & 0.0 (reference) & 0.0 (reference) & 0.0 (reference) & 0.0 (reference) \\
\hline Q2 & $-0.57(-1.22,0.07)$ & $-0.12(-0.69,0.46)$ & $-0.45(-1.03,0.14)$ & $-0.40(-0.98,0.19)$ & $-0.46(-1.02,0.09)$ & $1.07(0.49,1.65)$ \\
\hline Q3 & $-0.72(-1.31,-0.12)$ & $-0.57(-1.27,0.13)$ & $-0.15(-0.80,0.49)$ & $-0.65(-1.25,-0.06)$ & $-0.99(-1.57,-0.42)$ & $0.03(-0.61,0.67)$ \\
\hline Q4 & $-1.50(-2.17,-0.84)$ & $-0.53(-1.14,0.07)$ & $-0.42(-1.04,0.19)$ & $-2.05(-2.91,-1.20)$ & $-1.42(-2.69,-0.15)$ & $-1.86(-3.17,-0.56)$ \\
\hline Per Fourth & $-0.45(-0.66,-0.25)$ & $-0.20(-0.39,-0.004)$ & $-0.12(-0.31,0.07)$ & $-0.52(-0.76,-0.28)$ & $-0.49(-0.75,-0.23)$ & $-0.29(-0.58,-0.002)$ \\
\hline $\mathrm{p}$-value & $\leq 0.001$ & 0.04 & 0.23 & $\leq 0.001$ & $\leq 0.001$ & 0.05 \\
\hline \multicolumn{7}{|l|}{ Model 2} \\
\hline Q1 & 0.0 (reference) & 0.0 (reference) & 0.0 (reference) & 0.0 (reference) & 0.0 (reference) & 0.0 (reference) \\
\hline Q2 & $-0.77(-1.42,-0.13)$ & $-0.32(-0.89,0.26)$ & $-0.40(-0.98,0.18)$ & $-0.58(-1.17,0.003)$ & $-0.26(-0.81,0.29)$ & $-0.23(-0.85,0.39)$ \\
\hline Q3 & $-1.04(-1.63,-0.44)$ & $-0.93(-1.63,-0.23)$ & $0.13(-0.51,0.77)$ & $-0.95(-1.54,-0.36)$ & $-0.47(-1.05,0.11)$ & $-1.75(-2.46,-1.03)$ \\
\hline Q4 & $-2.02(-2.68,-1.35)$ & $-0.89(1.50,-0.29)$ & $-0.03(-0.65,0.58)$ & $-2.63(-3.48,-1.77)$ & $-1.64(-2.91,-0.38)$ & $-3.74(-5.07,-2.40)$ \\
\hline Per Fourth & $-0.62(-0.83,-0.41)$ & $-0.32(-0.51,-0.13)$ & $0.02(-0.18,0.21)$ & $-0.68(-0.92,-0.45)$ & $-0.32(-0.58,-0.06)$ & $-1.10(-1.41,-0.79)$ \\
\hline $\mathrm{p}$-value & $\leq 0.001$ & 0.001 & 0.88 & $\leq 0.001$ & 0.02 & $\leq 0.001$ \\
\hline \multicolumn{7}{|l|}{ Model 3} \\
\hline Q1 & 0.0 (reference) & 0.0 (reference) & 0.0 (reference) & 0.0 (reference) & 0.0 (reference) & 0.0 (reference) \\
\hline Q2 & $-0.85(-1.50,-0.19)$ & $-0.09(-0.67,0.50)$ & $-0.22(-0.81,0.38)$ & $-0.55(-1.15,0.05)$ & $-0.30(-0.86,0.26)$ & $-0.40(-1.04,0.25)$ \\
\hline Q3 & $-0.69(-1.31,-0.08)$ & $-0.69(-1.40,0.03)$ & $0.23(-0.43,0.88)$ & $-0.80(-1.41,-0.19)$ & $-0.13(-0.73,0.46)$ & $-1.57(-2.31,-0.84)$ \\
\hline Q4 & $-1.58(-2.27,-0.89)$ & $-0.37(-0.99,0.25)$ & $0.01(-0.62,0.64)$ & $-2.15(-3.03,-1.27)$ & $-1.15(-2.46,0.15)$ & $-3.70(-5.05,-2.34)$ \\
\hline Per Fourth & $-0.45(-0.66,-0.23)$ & $-0.16(-0.36,0.04)$ & $0.03(-0.17,0.23)$ & $-0.56(-0.80,-0.31)$ & $-0.15(-0.41,0.12)$ & $-1.01(-1.34,-0.69)$ \\
\hline $\mathrm{p}$ - value & $\leq 0.001$ & 0.12 & 0.75 & $\leq 0.001$ & 0.29 & $\leq 0.001$ \\
\hline
\end{tabular}

$\mathrm{FV}$, fruit and vegetables. FVJ, fruit, vegetables and juice.

Values represent mean difference (95\% CI) from reference category (Q1).

Model 1 unadjusted; Model 2 adjusted for age, country; Model 3 adjusted for age, country, cholesterol, BMI, height, physical activity, alcohol intake, education level, material conditions, smoking, diabetes and CHD history. 
Table 5. Logistic regression analysis of the association between hypertension (SBP $>=140 \mathrm{mmHg}$ ) and FV variables in the PRIME study

\begin{tabular}{|c|c|c|c|c|c|c|}
\hline & FVJ & Citrus fruit & Fruit juices & Other fruit & Cooked vegetables & Raw vegetables \\
\hline \multicolumn{7}{|l|}{ Model 1} \\
\hline Q1 & 1.0 (reference) & 1.0 (reference) & 1.0 (reference) & 1.0 (reference) & 1.0 (reference) & 1.0 (reference) \\
\hline Q2 & $0.92(0.82,1.04)$ & $0.86(0.77,0.95)$ & $0.87(0.79,0.97)$ & $0.87(0.78,0.97)$ & $1.01(0.92,1.12)$ & $0.93(0.84,1.04)$ \\
\hline Q3 & $0.82(0.76,0.92)$ & $0.89(0.79,1.01)$ & $0.94(0.84,1.06)$ & $0.89(0.80,0.99)$ & $1.04(0.94,1.15)$ & $0.77(0.69,0.87)$ \\
\hline Q4 & $0.79(0.70,0.59)$ & $0.85(0.77,0.95)$ & $1.02(0.91,1.13)$ & $0.70(0.60,0.82)$ & $0.93(0.73,1.17)$ & $0.55(0.43,0.71)$ \\
\hline Per Fourth & $0.93(0.89,0.96)$ & $0.96(0.92,0.99)$ & $1.00(0.97,1.04)$ & $0.92(0.88,0.96)$ & $1.01(0.96,1.06)$ & $0.86(0.81,0.90)$ \\
\hline p-value & $\leq 0.001$ & 0.01 & 0.96 & $\leq 0.001$ & 0.74 & $\leq 0.001$ \\
\hline \multicolumn{7}{|l|}{ Model 2} \\
\hline Q1 & 1.0 (reference) & 1.0 (reference) & 1.0 (reference) & 1.0 (reference) & 1.0 (reference) & 1.0 (reference) \\
\hline Q2 & $0.92(0.82,1.03)$ & $0.87(0.78,0.97)$ & $0.90(0.81,0.998)$ & $0.87(0.78,0.97)$ & $1.01(0.92,1.12)$ & $0.90(0.81,1.01)$ \\
\hline Q3 & $0.82(0.74,0.92)$ & $0.90(0.79,1.02)$ & $0.96(0.85,1.08)$ & $0.88(0.79,0.98)$ & $1.03(0.93,1.15)$ & $0.73(0.64,0.84)$ \\
\hline Q4 & $0.79(0.70,0.89)$ & $0.85(0.76,0.95)$ & $1.02(0.91,1.13)$ & $0.68(0.58,0.80)$ & $0.91(0.72,1.16)$ & $0.52(0.40,0.67)$ \\
\hline Per Fourth & $0.92(0.89,0.96)$ & $0.95(0.92,0.99)$ & $1.00(0.97,1.04)$ & $0.91(0.87,0.95)$ & $1.00(0.96,1.05)$ & $0.83(0.79,0.88)$ \\
\hline $\mathrm{p}$-value & $\leq 0.001$ & 0.01 & 0.92 & $\leq 0.001$ & 0.91 & $\leq 0.001$ \\
\hline \multicolumn{7}{|l|}{ Model 3} \\
\hline Q1 & 1.0 (reference) & 1.0 (reference) & 1.0 (reference) & 1.0 (reference) & 1.0 (reference) & 1.0 (reference) \\
\hline Q2 & $0.94(0.83,1.07)$ & $0.92(0.82,1.03)$ & $0.99(0.88,1.11)$ & $0.90(0.80,1.02)$ & $1.04(0.93,1.16)$ & $0.88(0.77,1.00)$ \\
\hline Q3 & $0.88(0.78,0.99)$ & $0.96(0.83,1.10)$ & $1.02(0.99,1.17)$ & $0.91(0.81,1.03)$ & $1.13(1.00,1.27)$ & $0.77(0.67,0.89)$ \\
\hline Q4 & $0.88(0.77,1.01)$ & $0.93(0.82,1.05)$ & $1.07(0.94,1.21)$ & $0.74(0.62,0.89)$ & $0.99(0.76,1.30)$ & $0.53(0.40,0.72)$ \\
\hline Per Fourth & $0.95(0.91,1.00)$ & $0.98(0.94,1.02)$ & $1.02(0.98,1.06)$ & $0.93(0.89,0.98)$ & $1.05(0.99,1.10)$ & $0.86(0.80,0.91)$ \\
\hline p- value & 0.03 & 0.32 & 0.30 & 0.01 & 0.11 & $\leq 0.001$ \\
\hline
\end{tabular}

FV, fruit and vegetables. FVJ, fruit, vegetables and juice. OR, Odds ratio. SBP, systolic blood pressure.

Values represent OR (95\% CI) for FV intake in comparison to Q1 (reference category).

Model 1 unadjusted; Model 2 adjusted for age, country; Model 3 adjusted for age, country, cholesterol, BMI, height, physical activity, alcohol intake, education level, material conditions, smoking, diabetes and CHD history. 


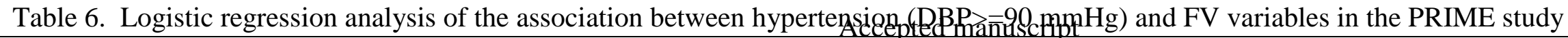

\begin{tabular}{|c|c|c|c|c|c|c|}
\hline & FVJ & Citrus fruit & Fruit juices & Other fruit & Cooked vegetables & Raw vegetables \\
\hline \multicolumn{7}{|l|}{ Model 1} \\
\hline Q1 & 1.0 (reference) & 1.0 (reference) & 1.0 (reference) & 1.0 (reference) & 1.0 (reference) & 1.0 (reference) \\
\hline Q2 & $0.89(0.79,1.01)$ & $0.94(0.84,1.04)$ & $0.93(0.83,1.04)$ & $0.95(0.85,1.07)$ & $0.98(0.88,1.08)$ & $1.15(1.03,1.28)$ \\
\hline Q3 & $0.86(0.77,0.96)$ & $0.87(0.76,0.99)$ & $0.99(0.87,1.11)$ & $0.87(0.78,0.98)$ & $0.92(0.83,1.03)$ & $0.93(0.82,1.05)$ \\
\hline Q4 & $0.79(0.70,0.90)$ & $0.91(0.81,1.02)$ & $1.03(0.92,1.16)$ & $0.69(0.59,0.82)$ & $0.78(0.61,1.01)$ & $0.72(0.55,0.94)$ \\
\hline Per Fourth & $0.93(0.89,0.97)$ & $0.97(0.93,1.00)$ & $1.01(0.97,1.05)$ & $0.91(0.87,0.95)$ & $0.95(0.90,1.00)$ & $0.93(0.88,0.98)$ \\
\hline p- value & $\leq 0.001$ & 0.07 & 0.67 & $\leq 0.001$ & 0.04 & 0.01 \\
\hline \multicolumn{7}{|l|}{ Model 2} \\
\hline Q1 & 1.0 (reference) & 1.0 (reference) & 1.0 (reference) & 1.0 (reference) & 1.0 (reference) & 1.0 (reference) \\
\hline Q2 & $0.87(0.77,0.98)$ & $0.91(0.82,1.02)$ & $0.93(0.84,1.05)$ & $0.93(0.83,1.04)$ & $1.00(0.90,1.11)$ & $0.97(0.86,1.09)$ \\
\hline Q3 & $0.83(0.74,0.93)$ & $0.83(0.73,0.95)$ & $1.02(0.91,1.16)$ & $0.84(0.75,0.94)$ & $0.98(0.88,1.10)$ & $0.74(0.64,0.85)$ \\
\hline Q4 & $0.74(0.65,0.84)$ & $0.87(0.78,0.98)$ & $1.08(0.96,1.21)$ & $0.64(0.54,0.76)$ & $0.76(0.59,0.98)$ & $0.57(0.43,0.75)$ \\
\hline Per Fourth & $0.91(0.87,0.95)$ & $0.95(0.92,0.99)$ & $1.03(0.99,1.06)$ & $0.89(0.85,0.93)$ & $0.97(0.92,0.02)$ & $0.84(0.79,0.89)$ \\
\hline p-value & $\leq 0.001$ & 0.01 & 0.19 & $\leq 0.001$ & 0.22 & $\leq 0.001$ \\
\hline \multicolumn{7}{|l|}{ Model 3} \\
\hline Q1 & 1.0 (reference) & 1.0 (reference) & 1.0 (reference) & 1.0 (reference) & 1.0 (reference) & 1.0 (reference) \\
\hline Q2 & $0.86(0.76,0.99)$ & $0.98(0.87,1.10)$ & $0.96(0.84,1.08)$ & $0.96(0.84,1.08)$ & $0.99(0.88,1.11)$ & $0.91(0.79,1.04)$ \\
\hline Q3 & $0.89(0.79,1.01)$ & $0.89(0.77,1.03)$ & $1.07(0.94,1.23)$ & $0.87(0.77,0.99)$ & $1.03(0.91,1.17)$ & $0.73(0.62,0.85)$ \\
\hline Q4 & $0.79(0.69,0.91)$ & $0.97(0.86,1.11)$ & $1.11(0.97,1.26)$ & $0.66(0.55,0.80)$ & $0.79(0.59,1.04)$ & $0.52(0.39,0.71)$ \\
\hline Per Fourth & $0.94(0.90,0.98)$ & $0.99(0.95,1.03)$ & $1.04(1.00,1.08)$ & $0.90(0.85,0.95)$ & $0.99(0.94,1.05)$ & $0.83(0.77,0.89)$ \\
\hline p-value & 0.001 & 0.46 & 0.08 & $\leq 0.001$ & 0.73 & $\leq 0.001$ \\
\hline
\end{tabular}

DBP, diastolic blood pressure, FV, fruit and vegetables. FVJ, fruit, vegetables and juice. OR, Odds ratio.

Values represent OR (95\% CI) for FV intake in comparison to Q1 (reference).

Model 1 unadjusted; Model 2 adjusted for age, country; Model 3 adjusted for age, country, cholesterol, BMI, height, physical activity, alcohol intake, education level, material conditions, smoking, diabetes and CHD history. 